\title{
Biochemical Effects of Carbohydrate Supplementation in a Simulated Competition of Short Terrestrial Duathlon
}

\author{
Renata Teixeira Mamus ${ }^{1}$, Maria Gisele dos Santos $^{2}$, Bill Campbell $^{3}, \&$ Richard Kreider $^{3}$ \\ Department of Physical Education, Dom Bosco Faculty ${ }^{1}$; Department of Physical Education, Federal University \\ of Paraná (UFPR), Curitiba, Paraná, Brazil ${ }^{2}$. Exercise and Sport Nutrition Lab, Baylor University, Waco TX ${ }^{3}$. \\ Address correspondence to rtmamus@yahoo.com.br.
}

Recieved November 8, 2005/Accepted November 8, 2006

\begin{abstract}
The purpose of the present study was to investigate the biochemical effects of carbohydrate supplementation in a simulated competition of short terrestrial duathlon. Ten duathletes participated in a simulated competition of short terrestrial duathlon 30 minutes after the ingestion of a $6 \%(30 \mathrm{~g} / 500 \mathrm{ml})$ maltodextrin solution (MALT) or a placebo (PLA). This solution was also ingested every 15 minutes during the competition $(12 \mathrm{~g} / 200 \mathrm{ml})$; and immediately after the competition $(18 \mathrm{~g} / 300 \mathrm{ml})$. Samples of blood were collected at 3 time points: 1$)$ at rest 1 hour before the beginning of the competition; 2) during the competition (approximately 1 hour and 45 minutes after the 1st collection); 3) immediately after the competition. Blood was analyzed for blood glucose, lactate, insulin and cortisol. Significant differences were observed in relation to blood glucose levels between MALT and PLA in the post-competition phase. There was also a significant difference in the lactate levels observed between MALT and PLA during the competition phase. Similarly, a significant difference in the cortisol concentrations during and after the competition phases (MALT and PLA) were observed. We conclude that maltodextrin supplementation appears to be beneficial during short terrestrial duathlon competition as evidenced by biochemical markers. Journal of the International Society of Sports Nutrition. 3(2): 6-11, 2006
\end{abstract}

Key-words: sports nutrition, carbohydrates; blood glucose; lactate; insulin; cortisol.

\section{INTRODUCTION}

The use of dietary interventions and the consumption of nutrients for the purpose of increasing performance are age-old practices. This is not surprising considering the highly competitive atmosphere that athletes engage in and considering their motivation to win. The willingness of athletes to experiment with scientifically sound nutritional interventions is a phenomenon that grows every day. While the use of nutritional supplements used by athletes is common, many facets relative to appropriate consumption of supplements and nutritional interventions still need to be discussed ${ }^{1}$.

Many studies have been investigating carbohydrate supplementation in relation to time of ingestion, frequency of ingestion, and concentration of the glucose solution ${ }^{2-4}$. In relation to long-duration exercise, it is well known that the primary fuel for long duration exercise is fat, although carbohydrate (glucose) is extremely important for fatty acid oxidation. Glucose, then, plays an important role for muscle performance, especially during relatively long and intense activities 5. As such, carbohydrate ingestion is recommended for athletes that participate in competitions that last an hour or more due to its rapid oxidation and its ability to be digested and absorbed quicker than proteins and lipids 6. Knowing that carbohydrate is the oxidizable fuel during high intense exercise, those athletes who train intensely or compete for consecutive days without consuming adequate amounts of carbohydrate are not able to maximize skeletal muscle glycogen storage and consequently are unable to perform at their best ${ }^{7}$.

Several studies have demonstrated that hepatic and muscular glycogen is important to maintain performance during exercise ${ }^{8,9}$. There is a correlation between initial glycogen levels and time of exercise duration when performing at a moderate intensity (75\% VO2max). It has also been shown that the 
relative contribution of skeletal muscle glycogen and glucose to energy production during exercise varies due to intensity and duration ${ }^{7}$. These investigations provide evidence to the importance of not only maximizing glycogen levels before an athletic event, but also highlight the importance of maintaining muscular and hepatic glycogen levels during an athletic event. The purpose of this study aimed at analyzing the biochemical effects of supplemental carbohydrate (in the form of maltodextrin) in a simulated competition of short terrestrial duathlon.

\section{METHODOLOGY}

Athletes from duathlon training centers were invited to participate in the study. All athletes that agreed to participate in the study were informed about the procedures involved in the investigation and signed a consent form that was approved by the Ethical Committee of the Federal University of Paraná (UFPR). Table 2 presents the descriptive characteristics of the duathletes that participated in the study.

Experimental Design. Supplementation was administered via a double-blind protocol. The athletes participated in the two sporting modalities (running, cycling) that comprise the short terrestrial duathlon. The duathlon consisted of 3 stages: an initial run stage $(5 \mathrm{Km})$, a cycling stage $(20 \mathrm{Km})$, and a second run stage $(2.5 \mathrm{Km})$ during a simulated competition where each participant received a liquid maltodextrin supplement or placebo. Supplementation was ingested before, during, and after the 3 stages. Blood samples were collected at rest 1 hour before the beginning of the competition; between the cycling stage and the second race stage (approximately 1 hour and 45 minutes after the 1st collection); and immediately after the competition. Glucose, lactate, insulin, and cortisol were later analyzed. The duration of the simulated race was one hour and fifteen minutes. Participants were assigned to each group based on their maximum $\mathrm{VO} 2$ max. Ten male duathletes from training centers of Curitiba (Pr), aged 17 to 35 years, participated in the study.

Instruments and Procedures. A modified Bruce protocol (ACSM, 2000) was administered to each participant to determine maximal oxygen consumption in order to randomize the athletes to the different groups. This test was administered during the week of the simulated competition. The treadmill used for the test was the ECAFIX (EG700X). Expired gases were analyzed via a PARVO MEDICS
TRUE MAX 2400. A physician was present during the maximum $\mathrm{VO} 2$ tests.

Supplementation. The athletes were divided into two groups (athletes were assigned to each group based on their maximum VO2 max.): MALT $(n=5)$ and PLA $(\mathrm{n}=5)$. MALT ingested maltodextrin (D.N.A, advanced nutrition design) and PLA ingested a placebo (Clight, extracted through pineapple juice). Also, $0.25 \mathrm{~g} / 1$ of pineapple juice (Clight) was added to the maltodextrin supplement for the purposes of taste blinding. The MALT and PLA drinks were ingested at 3 different time points throughout the simulated competition: 30 minutes before the competition, $\quad(6 \%$ solution of carbohydrate $[30 \mathrm{~g} / \mathrm{CHO} / 500 \mathrm{ml}])$; every 15 minutes during the competition $(6 \%$ solution of carbohydrate $[12 \mathrm{~g} / \mathrm{CHO} / 200 \mathrm{ml}])$; and immediately after the competition $(6 \%$ solution of carbohydrate $[18 \mathrm{~g} / \mathrm{CHO} / 300 \mathrm{ml}])$.

Biochemical Analyses. All biochemical analyses were completed at the Service of Clinical Analyses from the Federal University Hospital of Paraná (SAC/UFPR). Blood samples were obtained through venous collection (needle and syringe) in the antecubital vein of each duathlete at 3 different time points: at rest 1 hour before the beginning of the competition; between the cycling stage and the second race stage (approximately 1 hour and 45 minutes after the 1st collection); and immediately after the competition. Each sample of blood was separated in two tubes: one containing fluoride $(4 \mathrm{ml})$ for glucose and lactate analysis; and the other $(8 \mathrm{ml})$ containing a gel sifter for the analysis of insulin and cortisol. The concentration of circulating glucose was accomplished via the Glucose Hexokinase II (GLU H II) method through the Glucose Hexokinase II Kit and ADVIA 1650 reagents (Bayer). Serum lactate was analyzed by the colorimeter enzymatic method as described by Engle \& Jones 10. Insulin levels were determined by immunoassay and immunometric assay methods on an IMMULITE 2000 automated analyzer.

Statistics. STATISTICA (version 5.5) was used for data analysis. The variables were analyzed through the non-parametric Friedman Test which identified any significant differences in the same group throughout the 3 stages of the simulated competition; the Kruskal-Wallis Test was also used to identify if there were significant differences between the different groups. For the purpose of completing the analysis of the Friedman and Kruskal-Wallis tests, a test of multiple comparisons was used to identify 
where the significance existed if differences were observed. The level of significance was set at $p$ $<0.05$.

\section{RESULTS}

Table 1 presents the descriptive characteristics of the duathletes that participated in the study. All data is expressed as means \pm standard deviation. Descriptive values relative to glucose, insulin, lactate, and cortisol are shown in table 2 .

Table 1. Subject characteristics.

\begin{tabular}{l|l}
\hline \multicolumn{1}{c|}{ VARIABLE } & ATHLETES $(\mathrm{n}=10)$ \\
\hline Age (years) & $27.11 \pm 8.75$ \\
MALT VO $\mathrm{VO}_{2}$ máx $\left(\mathrm{ml} \cdot \mathrm{Kg}^{-1} \cdot \mathrm{min}^{-1}\right)$ & $62.14 \pm 7.83$ \\
PLA VO $\mathrm{VO}_{2}$ máx $\left(\mathrm{ml} \cdot \mathrm{Kg}^{-1} \cdot \mathrm{min}^{-1}\right)$ & $62.14 \pm 6.86$ \\
\hline
\end{tabular}

Table 2. Glucose, insulin, lactate, and cortisol concentrations at each stage of competition.

\begin{tabular}{ccccc}
\hline Stage & $\begin{array}{c}\text { GLUCOSE } \\
(\mathrm{mmol} / \mathrm{L})\end{array}$ & $\begin{array}{c}\text { INSULIN } \\
(\mathrm{UI} / \mathrm{mL})\end{array}$ & $\begin{array}{c}\text { LACTATE } \\
(\mathrm{mmol} / \mathrm{L})\end{array}$ & $\begin{array}{c}\text { CORT } \\
(\mathrm{g} / \mathrm{dL})\end{array}$ \\
\hline Pre & & & & \\
Malt & $5.4 \pm 0.3$ & $9.8 \pm 5.9$ & $1.4 \pm 0.2$ & $13.1 \pm 2.8$ \\
Pla & $5.2 \pm 0.4$ & $11.3 \pm 10.1$ & $2.0 \pm 1.1$ & $13.1 \pm 3.8$ \\
& & & & \\
During & & & & \\
Malt & $6.4 \pm 0.5$ & $7.5 \pm 2.4$ & $10.3 \pm 2.9$ & $23.6 \pm 5.8$ \\
Pla & $5.8 \pm 1.2$ & $6.5 \pm 1.0$ & $9.6 \pm 1.0$ & $22.4 \pm 5.6$ \\
& & & & \\
Post & & & & \\
Malt & $7.0 \pm 0.4 * \dagger$ & $8.1 \pm 2.1$ & $5.2 \pm 1.2 *$ & $20.8 \pm 5.2^{*}$ \\
Pla & $4.7 \pm 0.7 * \dagger$ & $6.8 \pm 4.6$ & $6.1 \pm 2.8 *$ & $25.1 \pm 4.3^{*}$ \\
& & & & \\
\hline
\end{tabular}

Values are mean $\pm \mathrm{SD}$. MALT $=$ maltodextrin group; PLA $=$ placebo group. Units of Measure: CORT = cortisol. * significant differences among the phases of the same group; $\mathrm{p}<0.05 ; \dagger$ significant differences among different groups; $\mathrm{p}<0.05$.

As shown in table 2, MALT presented a significant difference $(\mathrm{p}<.001)$ in the blood glucose concentrations between the pre- and post-competition phases, indicating an increase of blood glucose concentrations during the competition. PLA presented a significant decrease $(p<0.05)$ in blood glucose levels from pre to post-competition. Comparing groups MALT and PLA relative to blood glucose, no significant differences were found between the pre-and during phases of the competition; however, a significant difference $(p<0.05)$ in the post-competition phase was found.
No significant differences were observed for insulin concentrations during the competition phases among the different groups. Significant time differences $(\mathrm{p}<0.05)$ were observed for MALT and PLA in the post-competition phase for lactate concentrations. However, when the levels of the lactate among the 2 groups were compared, there were no significant differences in any phase of the competition.

A significant decrease $(p<0.05)$ in cortisol concentrations was observed for MALT in the postcompetition phase. Conversely, there was a significant increase $(p<0.05)$ for cortisol concentrations in the PLA group in the postcompetition phase. No significant differences were observed between the groups relative to cortisol levels.

\section{DISCUSSION}

Blood Glucose and Insulin Concentrations. A significant increase in blood glucose levels was observed in those duathletes ingesting maltodextrin in comparison to those ingesting a placebo. Those duathletes ingesting a placebo experienced a significant decrease in blood glucose in the postcompetition phase as compared to the precompetition phase. These findings have also been reported in other investigations ${ }^{11-15}$. The observed increase in blood glucose levels in MALT after the competition is primarily due to the ingestion of $\mathrm{CHO}$ (maltodextrin) before and during the competition, which exerts an additional effect on physical performance when compared to ingesting $\mathrm{CHO}$ at only one time point (pre- or during competition) ${ }^{12}$. Attaining elevated blood glucose concentrations is indispensable for physical performance, given that high blood glucose concentrations favor glycolysis during physical activity. Conversely, a decrease in blood glucose concentrations can lead to fatigue during competition ${ }^{13}$.

Maltodextrin ingestion leading to increased levels of circulating glucose has been shown to prevent hypoglycemia while allowing hepatic glucose production to be uninhibited ${ }^{15}$. Preventing hypoglycemia and increasing skeletal muscle glycogen stores allows for prolonged exertion and delays the onset of fatigue. This delay in fatigue is due mainly to the prevention of the decline of the blood glucose concentration, which is highly oxidized during the latter stages of the competition 9,20 . The findings in the present study are in agreement with other investigations relative to 
increases in blood glucose levels after maltodextrin ingestion ${ }^{9,13,14}$.

Insulin concentrations did not elevate during the simulated race in either group. This finding is consistent with other studies which supplemented athletes with carbohydrate $(6 \%$ glucose $)$ during exercise ${ }^{15,16}$. Even with the increase in blood glucose concentrations resulting from maltodextrin ingestion, insulin concentrations tend to decrease during physical exertion. The present study demonstrated this decrease in insulin secretion despite maltodextrin ingestion. This is partially explained by two factors: a) an increased activity of GLUT 4 (translocation to the cell membrane); b) an increase of blood flow to the working muscle which allows for greater uptake of blood glucose. Each of these factors supports the actions of insulin, thereby reducing the quantity needed for glucose disposal ${ }^{7}$.

Concentrations of Serum Lactate. In the present study we found significant differences in lactate concentrations over time in MALT and PLA, with the post-competition levels being significantly elevated as compared to baseline levels. In both of these groups, the lactate levels increased during the competition and decreased in the post-competition phase. Other studies have reported significant differences among groups that ingested maltodextrin and placebo where the lactate concentrations were higher during the competition in the group ingesting maltodextrin ${ }^{26}$. In the present study, an increase in lactate concentration can be observed during the competition, although, there was no significant difference found between the groups ingesting maltodextrin or placebo. In general, the lactate concentration increases soon after the beginning of physical exertion and can be maintained until the end of physical activity ${ }^{27}$. The importance of lactate production during physical exertion is due to the fact that it serves as a glucose substrate via hepatic gluconeogenesis. Our findings relative to the elevated levels of blood lactate in the MALT and PLA groups is supported in other investigations utilizing 6\% carbohydrate (maltodextrin) solutions $9,13,15,17$. Another result obtained in this study was the finding that there were no significant differences among the 2 groups relative to lactate concentrations. This observation supports the findings of other investigations that compared different treatments with $\mathrm{CHO}$ and placebo ${ }^{8,9,15}$.

Cortisol Concentrations. The cortisol levels increased during the competition stage in both groups. In the post-competition phase, there was a decrease in cortisol concentrations in the MALT group. Conversely, cortisol concentrations increased in the post-competition assessment for the PLA group. Cortisol is a hormone that usually increases during physical exertion, when the levels of endogenous carbohydrate significantly decrease 7 . Others 28 have reported changes in cortisol concentrations at different intensity levels in athletes completing several one-hour exercise sessions. In this study, each test was accomplished with a constant intensity between $40-80 \%$ of VO2 max. It was reported that an exercise intensity of $40 \%$ of VO2 max caused a decrease in cortisol levels, while an exercise intensity of $80 \%$ of $\mathrm{VO} 2$ max resulted in high elevations of the hormone. In the present study, the intensity of exercise was moderate at $79 \%$ of VO2 max.

Also, cortisol concentrations have been shown to increase significantly during a prolonged competition, demonstrating dramatic elevations in hypoglycemia levels ${ }^{25}$. However, with the ingestion of $\mathrm{CHO}$ (maltodextrin) the increased concentrations of cortisol can be lessened as compared to placebo ingestion ${ }^{19}$. This observation corresponds with the decrease in cortisol levels observed in the MALT group, confirming the fact that maltodextrin ingestion softens the increase of cortisol levels after an intense competition as compared to placebo ingestion. Other studies ${ }^{28}$ have reported a decrease in cortisol levels after ingesting $60 \mathrm{~g}$ of maltodextrin per hour during physical exertion. However, when the athletes ingested an inferior amount of maltodextrin $(13 \mathrm{~g} / \mathrm{h})$ there were no changes in the cortisol levels. In addition, other investigations have demonstrated a fall in cortisol concentrations during duathalon-type events in those athletes that ingested solutions with maltodextrin ${ }^{15,18}$. When comparing the 2 groups in the present study, no significant difference was observed between the pre and during-competition phases. This finding is in agreement with other investigations ${ }^{8,14}$.

\section{CONCLUSIONS}

Results obtained in the present study indicate the following conclusions:

A) Maltodextrin supplementation may enhance performance during a short terrestrial duathlon. This is based on the biochemical evidence which indicated an elevation of blood glucose and insulin; and a decrease in lactate levels and cortisol. These biochemical effects during the competition are important markers of optimizing physical performance, for an increase in blood 
glucose and a decrease in lactate levels are associated with delaying the onset of fatigue; B) The benefit of maltodextrin supplementation can be explained mainly by the maintenance of high levels of blood glucose, avoiding the likely performance decrements associated with hypoglycemia. In addition, high levels of blood glucose serve to restore depleted levels of glycogen, which also serve to delay muscular fatigue and improve endurance performance.

It can be concluded that the ingestion of a $6 \%$ solution of maltodextrin during a short terrestrial duathlon causes significant alterations in the plasma concentrations of glucose, lactate and plasmatic cortisol, which are considered the main biochemical markers that can delay fatigue during a competition. These findings corroborate previous results obtained in other studies accomplished with cyclists, marathon runners, and triathletes, confirming the benefits of the maltodextrin supplementation, through biochemical indices, making the findings of the present study eligible to be applied to the training routines and nutritional plans of duathletes. Further research is needed investigating the biochemical effects of ingesting different carbohydrate types during a short terrestrial duathlon event.

\section{REFERENCES}

1. Correia, M.I.T.D. Nutrição, Esporte e Saúde. Belo Horizonte: Health, 1996.

2. Ivy, J.L. et al. Muscle glycogen synthesis after exercise: effect of time of carbohydrate ingestion. J Appl Physiol . 64: 1480-1485, 1988.

3. Jentjens, R.L.P.G. et al. Addition of protein and amino acids to carbohydrates does not enhance postexercise. J Appl Physiol . 91: 839-846, 2001.

4. PiehL, A.K., Soderlund, K. and Hultman, E. Muscle glycogen resynthesis rate in humans after supplementation of drinks containing carbohydrates with low and high molecular masses. Eur J Appl Physiol . 81: 346-351, 2000.

5. Wolinsky I., and Hickson J. J. F. Nutrição no exercício e no esporte. 2nd ed., São Paulo: Roca, 1996.

6. Jacobs, K.A. and Sherman, W.M. The efficacy of carbohydrate supplementation and chronic high-carbohydrate diets for improving endurance performance. Int J Sport Nutr . 9(1): 92-115, 1999.

7. Wilmore, J.H. and Costill, D.L. Fisiologia do Esporte e do Exercício. São Paulo: Manole, 2001.

8. Mcconell, G.K. et al. Effect of carbohydrate ingestion on glucose kinetics and muscle metabolism during intense endurance exercise. J Appl Physiol. 89: 1690-1698, 2000.

9. Andrews, J.L. et al. Carbohydrate loading and supplementation in endurance-trained women runners. J Appl Physiol .95: 584-590, 2003.

10. Engle, P. C.; Jones, J. B. Causes and elimination of erratic blanks in enzymatic metabolite assays involving the use of NAD in alkaline hydrazine buffers: improved conditions for the assay of L-glutamate, L-lactate, and other metabolites. Analytical Biochemistry, v. 88, p. 475-84, 1978.

11. Powers, S.K. and Howley, E.T. Exercise Physiology: theory and application to fitness and performance. $4^{\text {a }}$ ed. New York: McGraw-Hill, 2001.

12. Wright, D.A. et al. Carbohydrate feedings before, during or in combination improve cycling performance. J Appl Physiol . 71: 1082-1088, 1991.

13. Bowtell, J.L. et al. Effect of different carbohydrate drinks on whole body carbohydrate storage after exhaustive exercise. J Appl Physiol. 88: 1529-1536, 2000.

14. Wong, S.H.,Williams, C. and Adams, N. Effects of ingesting a large volume of carbohydrate-electrolyte solution on rehydration during recovery and subsequent exercise capacity. Int J Sports Nutr . 10(4):234-240, 2000.

15. Angus, D.J. et al. Effect of carbohydrate ingestion on glucose kinetics during exercise in the heat. J Appl Physiol. 90: 601-605, 2001.

16. Wallis, GA, Dawson R, Achten J, Webber J, Jeukendrup AE. Metabolic response to carbohydrate ingestion during exercise in males and females. Am J Physiol Endocrinol Metab. 2006 290(4):E708-15.

17. Utter, A.C. et al. Effect of carbohydrate ingestion on ratings of perceived exertion during a marathon. Med Sci Sports Exerc. 34(11): 1779-1784, 2002.

18. Volek, J. S. Influence of Nutrition on Responses to Resistance Training. Med Sci Sports Exerc . 36 (4): 689-696, 2004.

19. Grenn, K. et al. Carbohydrate supplementation and exerciseinduced changes in T-lymphocyte function. J Appl Physiol. 95: 12161223, 2003.

20. Anderson, G.H. et al. Inverse association between the effect of carbohydrate on blood glucose and subsequente short-term food intake in young men. Am J Clin Nutr. 76(50): 1023-1030, 2002.

21. Maclaren, D.P.M. and Close, G.L. Effect of carbohydrate supplementation on simulated exercise of rugby league referees. Ergonomics. 43(10): 1528-1537, 2000.

22. Ivy, J.L. et al. Early postexercise muscle glycogen recovery is enhaced with a carbohydrate-protein supplement. J Appl Physiol . 93: 1337-1344, 2002.22

23. Hayashi, T. et al. Exercise regulation of glucose transport skeletal muscle. Am J Physiol 1997, 273: E1039-E1051, 1997.

24. Davis, J. M, and Brown, A. S. Carbohydrates, Hormones and Endurance Performance. Sports Science Exchange . 14 (1): 1-4, 2001.

25. Kraemer, W.J. et al. Hormonal responses to consecutive days of heavy-resistance with or without nutritional supplementation. J Appl Physiol. 85(4): 1544-1555, 1998. 
26. Jentjens, R.L.P.G., Venables, M.C., Jeukendrup, A.E. Oxidation of exogenous glucose, sucrose and maltose during prolonged cycling exercise. J Appl Physiol . 96: 1285-1291, 2004.

27. Lancha, A.H. Nutrição e metabolismo aplicados à atividade motora. São Paulo: Atheneu, 2002.

28. Murray, R. et al. Responses to varying rates of carbohydrate ingestion during exercise. Med Sci Sports Exerc 1991, 23: 713-718, 1991. 